\title{
Long-Range Coulomb Interaction and the Crossover between Quantum and Shot Noise in Diffusive Conductors
}

\author{
K. E. Nagaev \\ Institute of Radioengineering and Electronics, Russian Academy of Science, Mokhovaya 11, Moscow 103907, Russia
}

\begin{abstract}
Frequency-dependent nonequilibrium noise in quantum-coherent diffusive conductors is calculated with account taken of long-range Coulomb interaction. For long and narrow contacts with strong external screening the crossover between quantum and shot noise takes place at frequencies much smaller than the voltage drop across the contact. We also show that under certain frequency limitations, the semiclassical and quantum-coherent approaches to shot noise are mathematically equivalent.
\end{abstract}

PACS numbers: 72.70.+m, 73.23.-b, 73.50.Td, 73.23.Ps

\section{INTRODUCTION}

Recently, the shot noise in mesoscopic contacts became a subject of extensive study (see recent book 1 and review2). The $1 / 3$ shot noise suppression in diffusive metal contacts was obtained almost simultaneously in 1992 using the quantum-coherent 3 and semiclassical 14 approaches. Since then it was disputed whether both approaches addressed the same effect. For example, de Jong and Beenakker2 suggested that quantum coherence is not required for the 1/3 suppression, while Landauer $\mathbf{\theta}_{\text {argued }}$ that semiclassical and quantum-coherent effects are different and $1 / 3$ is just a numerical coincidence. Although very important properties of shot noise like universality 6 and exchange effect in multiterminal contacts 7 originally obtained within the quantum-coherent approach were then rederived using the semiclassical approach, 1 a direct proof of equivalence of these approaches is still absent. An important step in this direction was very recently made by Gramespacher and Büttiker, who introduced effective local distribution function for a phase-coherent nonequilibrium conductor and expressed the current fluctuations at local tunnel probes attached to it in terms of this function.9

A related problem is the frequency dependence of nonequilibrium noise in diffusive contacts. This dependence is affected by quantum effects and effects of self-consistent electrical field. While the former effects were successfully described in terms of quantum-coherent formula for the shot noise, 10 the latter presented a certain difficulty for this approach (see 11 for a more detailed discussion of this issue). Although particular results were obtained for nonequilibrium charge fluctuations in quantum point contacts and chaotic cavities in terms of discrete capacitances, 12 they could not be extended to metallic diffusive conductors, for which the potential needs to be treated as a field. On the contrarv, the semiclassical approach provides an easy treatment of long-range electron-electron interaction 13.14 yet rules out the description of quantum effects.

This paper is intended to some extent to bridge the gap between the phase-coherent and semiclassical approaches. We present a method for calculating frequency-dependent shot noise in a phase-coherent diffusive metallic conductor with account taken of self-consistent electrical field. This method is an extension of Keldysh Green's function formalism used by Altshuler et al. 15 to the case of interacting electrons 16 We show that phase-coherent equations may be written in the form very similar to the semiclassical ones, so that both approaches are mathematically equivalent at sufficiently low frequencies. As an illustration of this method, we calculate quantum noise in nonequilibrium contacts in the presence of a strong external screening. 


\section{BASIC EQUATIONS}

To calculate the noise, we make use of Keldysh diagrammatic technique, which is valid not only at finite temperatures, but also for nonequilibrium systems. The spectral density of noise is calculated as the Fourier transform of symmetrized current-current correlator

$$
S_{\alpha \beta}\left(\mathbf{r}_{1}, \mathbf{r}_{2}, \omega\right)=\int d\left(t_{1}-t_{2}\right) \exp \left[i \omega\left(t_{1}-t_{2}\right)\right]\left\langle\left\langle\delta \hat{j}_{\alpha}\left(\mathbf{r}_{1}, t_{1}\right) \delta \hat{j}_{\beta}\left(\mathbf{r}_{2}, t_{2}\right)+\delta \hat{j}_{\beta}\left(\mathbf{r}_{2}, t_{2}\right) \delta \hat{j}_{\alpha}\left(\mathbf{r}_{1}, t_{1}\right)\right\rangle\right\rangle
$$

where the double angular brackets dengte quantum-mechanical and statistical averaging and $\delta \hat{\mathbf{j}}=$ $\hat{\mathbf{j}}-\langle\langle\hat{\mathbf{j}}\rangle\rangle$. In terms of Keldysh contour, 17 this expression may be written in the form

$$
\begin{gathered}
S_{\alpha \beta}\left(\mathbf{r}_{1}, \mathbf{r}_{2}, \omega\right)=\frac{e^{2}}{4 m}\left(\frac{\partial}{\partial \mathbf{r}_{1 \alpha}}-\frac{\partial}{\partial \mathbf{r}_{1 \alpha}^{\prime}}\right)\left(\frac{\partial}{\partial \mathbf{r}_{2 \beta}}-\frac{\partial}{\partial \mathbf{r}_{2 \beta}^{\prime}}\right) \\
\times \int d\left(t_{1}-t_{2}\right) \exp \left[i \omega\left(t_{1}-t_{2}\right)\right]\left\langle G_{2121}^{I I}\left(\mathbf{r}_{1}, t_{1}+\delta ; \mathbf{r}_{2}, t_{2}-\delta ; \mathbf{r}_{1}^{\prime}, t_{1}-\delta ; \mathbf{r}_{2}^{\prime}, t_{2}+\delta\right)\right. \\
+G_{1212}^{I I}\left(\mathbf{r}_{1}, t_{1}-\delta ; \mathbf{r}_{2}, t_{2}+\delta ; \mathbf{r}_{1}^{\prime}, t_{1}+\delta ; \mathbf{r}_{2}^{\prime}, t_{2}-\delta\right)-G_{22}\left(\mathbf{r}_{1}, t_{1}+\delta ; \mathbf{r}_{1}^{\prime}, t_{1}-\delta\right) G_{11}\left(\mathbf{r}_{2}, t_{2}-\delta ; \mathbf{r}_{2}^{\prime}, t_{2}+\delta\right) \\
\left.-G_{11}\left(\mathbf{r}_{1}, t_{1}-\delta ; \mathbf{r}_{1}^{\prime}, t_{1}+\delta\right) G_{22}\left(\mathbf{r}_{2}, t_{2}+\delta ; \mathbf{r}_{2}^{\prime}, t_{2}-\delta\right)\right\rangle,
\end{gathered}
$$

where $\mathbf{r}_{1}^{\prime} \rightarrow \mathbf{r}_{1}$ and $\mathbf{r}_{2}^{\prime} \rightarrow \mathbf{r}_{2}$. The two-particle Green's functions are given by the expression

$$
G_{i j k l}^{I I}\left(\mathbf{r}_{1} t_{1} ; \mathbf{r}_{2} t_{2} ; \mathbf{r}_{3} t_{3} ; \mathbf{r}_{4} t_{4}\right)=\left\langle\left\langle T_{c} \psi\left(\mathbf{r}_{1}, t_{1 i}\right) \psi\left(\mathbf{r}_{2}, t_{2 j}\right) \psi^{+}\left(\mathbf{r}_{3}, t_{3 k}\right) \psi^{+}\left(\mathbf{r}_{4}, t_{4 l}\right)\right\rangle\right\rangle
$$

and $T_{c}$ is the operator of time ordering on the two-branch Keldysh temporal contour. Indices $i, j, k, l$ denote the number of contour branch. It is equal to 1 if the branch goes from $-\infty$ to $+\infty$ and 2 if it goes from $+\infty$ to $-\infty$. The points of the first branch are considered as preceding to the points of the second branch.
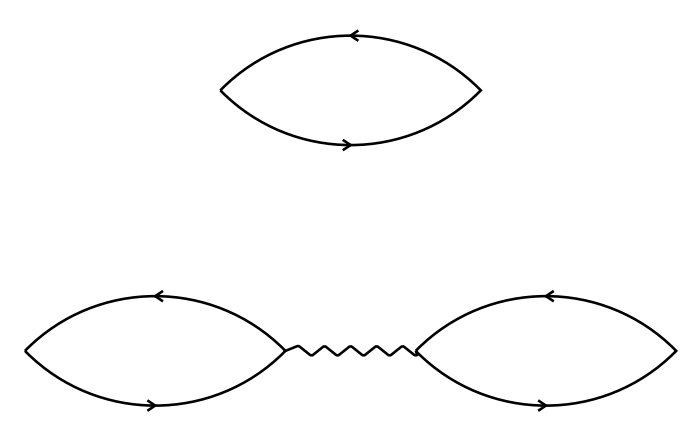

FIG. 1. Basic diagrams for the spectral density of noise.

The diagrams contributing to $S_{\alpha \beta}$ in the random-phase approximation are shown in Fig. 1. Each vertex corresponds to one of the branches of the Keldysh contour, solid lines correspond to singleelectron Green's functions

$$
G_{i j}\left(\mathbf{r}_{1} t_{1}, \mathbf{r}_{2} t_{2}\right)=-i\left\langle\left\langle T_{c} \psi\left(\mathbf{r}_{1} t_{1 i}\right) \psi^{+}\left(\mathbf{r}_{2} t_{2 j}\right)\right\rangle\right\rangle
$$

and the wavy line corresponds to the potential of screened Coulomb interaction $V_{i j}$. The summation over the Keldysh indices is performed at the inner vertices. The diagrams shown in Fig. 1 have 
a common property that the small momentum $q_{t r}$ of the order of inverse contact length $L^{-1}$ is transferred from the left to the right through a single channel, i.e. by a single interaction line or a pair of lines describing electron-hole propagation. The diagrams that correspond to a redistribution of this momentum among different interaction lines or interaction lines and electron-hole pairs involve additional integrations over the momentum transfer and contain a small factor $1 / p_{F} l_{i m p}$, where $p_{F}$ is the Fermi momentum and $l_{i m p}$ is the elastic mean free path of electron.18

The four functions $G_{i j}$ may be expressed in terms of the retarded Green's function $G^{R}$, advanced Green's function $G^{A}$, and the electron correlator $G^{K}$ :

$$
\begin{array}{ll}
G_{11}=\left(G^{R}+G^{A}+G^{K}\right) / 2, & G_{12}=\left(-G^{R}+G^{A}+G^{K}\right) / 2, \\
G_{21}=\left(G^{R}-G^{A}+G^{K}\right) / 2, & G_{22}=\left(-G^{R}-G^{A}+G^{K}\right) / 2 .
\end{array}
$$

Similarly, the four potentials $V_{i j}$ may be expressed in terms of the three independent quantities

$$
\begin{array}{cc}
V_{11}=\left(V^{R}+V^{A}+V^{K}\right) / 2, & V_{12}=\left(V^{R}-V^{A}-V^{K}\right) / 2, \\
V_{21}=\left(-V^{R}+V^{A}-V^{K}\right) / 2, & V_{22}=\left(-V^{R}-V^{A}+V^{K}\right) / 2 .
\end{array}
$$

On this substitution, the spectral density assumes the form

$$
\begin{gathered}
S_{\alpha \beta}\left(\mathbf{r}_{1}, \mathbf{r}_{2}, \omega\right)=S_{\alpha \beta}^{(0)}-\frac{i}{4} \int d^{3} r_{3} \int d^{3} r_{4}\left[Q_{\alpha}\left(\mathbf{r}_{1}, \mathbf{r}_{3}, \omega\right) V^{R}\left(\mathbf{r}_{3}, \mathbf{r}_{4}, \omega\right) P_{\beta}\left(\mathbf{r}_{2}, \mathbf{r}_{4},-\omega\right)\right. \\
\left.+P_{\alpha}\left(\mathbf{r}_{1}, \mathbf{r}_{3}, \omega\right) V^{A}\left(\mathbf{r}_{3}, \mathbf{r}_{4}, \omega\right) Q_{\beta}\left(\mathbf{r}_{2}, \mathbf{r}_{4},-\omega\right)+Q_{\alpha}\left(\mathbf{r}_{1}, \mathbf{r}_{3}, \omega\right) V^{K}\left(\mathbf{r}_{3}, \mathbf{r}_{4}, \omega\right) Q_{\beta}\left(\mathbf{r}_{2}, \mathbf{r}_{4},-\omega\right)\right] .
\end{gathered}
$$

The quantity

$$
\begin{aligned}
S_{\alpha \beta}^{(0)}\left(\mathbf{r}_{1}, \mathbf{r}_{2}, \omega\right) & =-\frac{1}{16 \pi} \frac{e^{2}}{m^{2}}\left(\frac{\partial}{\partial \mathbf{r}_{1 \alpha}}-\frac{\partial}{\partial \mathbf{r}_{1 \alpha}^{\prime}}\right)\left(\frac{\partial}{\partial \mathbf{r}_{2 \beta}}-\frac{\partial}{\partial \mathbf{r}_{2 \beta}^{\prime}}\right) \int d \varepsilon\left\langle G^{R}\left(\mathbf{r}_{1}^{\prime}, \mathbf{r}_{2}, \varepsilon+\omega\right) G^{A}\left(\mathbf{r}_{2}^{\prime}, \mathbf{r}_{1}, \varepsilon\right)\right. \\
& \left.+G^{A}\left(\mathbf{r}_{1}^{\prime}, \mathbf{r}_{2}, \varepsilon+\omega\right) G^{R}\left(\mathbf{r}_{2}^{\prime}, \mathbf{r}_{1}, \varepsilon\right)+G^{K}\left(\mathbf{r}_{1}^{\prime}, \mathbf{r}_{2}, \varepsilon+\omega\right) G^{K}\left(\mathbf{r}_{2}^{\prime}, \mathbf{r}_{1}, \varepsilon\right)\right\rangle
\end{aligned}
$$

is the spectral density of noise of noninteracting electrons, and

$$
\begin{gathered}
P_{\alpha}\left(\mathbf{r}_{1}, \mathbf{r}_{2}, \omega\right)=-\frac{i e}{2 m}\left(\frac{\partial}{\partial \mathbf{r}_{1 \alpha}}-\frac{\partial}{\partial \mathbf{r}_{1 \alpha}^{\prime}}\right) \int \frac{d \varepsilon}{2 \pi}\left\langle G^{R}\left(\mathbf{r}_{1}^{\prime}, \mathbf{r}_{2}, \varepsilon+\omega\right) G^{A}\left(\mathbf{r}_{2}^{\prime}, \mathbf{r}_{1}, \varepsilon\right)\right. \\
\left.+G^{A}\left(\mathbf{r}_{1}^{\prime}, \mathbf{r}_{2}, \varepsilon+\omega\right) G^{R}\left(\mathbf{r}_{2}^{\prime}, \mathbf{r}_{1}, \varepsilon\right)+G^{K}\left(\mathbf{r}_{1}^{\prime}, \mathbf{r}_{2}, \varepsilon+\omega\right) G^{K}\left(\mathbf{r}_{2}^{\prime}, \mathbf{r}_{1}, \varepsilon\right)\right\rangle
\end{gathered}
$$

is the current - density correlator of noninteracting electrons. Single angular brackets denote impurity averaging. Both quantities have a similar structure, i.e. they contain an even number of correlators $G^{K}$. In contrast to this, the quantity

$$
\begin{gathered}
Q_{\alpha}\left(\mathbf{r}_{1}, \mathbf{r}_{2}, \omega\right)=-\frac{i e}{2 m}\left(\frac{\partial}{\partial \mathbf{r}_{1 \alpha}}-\frac{\partial}{\partial \mathbf{r}_{1 \alpha}^{\prime}}\right) \int \frac{d \varepsilon}{2 \pi}\left\langle G^{K}\left(\mathbf{r}_{1}^{\prime}, \mathbf{r}_{2}, \varepsilon+\omega\right) G^{A}\left(\mathbf{r}_{2}^{\prime}, \mathbf{r}_{1}, \varepsilon\right)\right. \\
\left.+G^{R}\left(\mathbf{r}_{1}^{\prime}, \mathbf{r}_{2}, \varepsilon+\omega\right) G^{K}\left(\mathbf{r}_{2}^{\prime}, \mathbf{r}_{1}, \varepsilon\right)\right\rangle
\end{gathered}
$$

contains only one $G^{K}$ and presents the current response to the external electric potential. Hence the first two terms in (3) have a simple physical meaning: they represent correlations between the current fluctuations caused directly by random motion of noninteracting electrons and the current fluctuations induced by density fluctuations of noninteracting electrons through the mediation of electric field. As will be shown below, the quantity $V^{K}$ represents a correlator of electric potentials, and the last term in (3) presents a correlator of currents induced by their fluctuations. 


\section{EVALUATION OF DIAGRAMS}

The key point in calculating the correlators of noninteracting electrons and their responses is the impurity averaging of the corresponding expressions. We start from the expressions for impurityaveraged single-electron Green's functions. The retarded and advanced Green's functions are given by expressions

$$
G^{R}\left(\mathbf{r}_{1}-\mathbf{r}_{2}\right)=\int \frac{d^{3} p}{(2 \pi)^{3}} \frac{\exp \left[i \mathbf{p}\left(\mathbf{r}_{1}-\mathbf{r}_{2}\right)\right]}{\varepsilon-\xi-e \phi-i / 2 \tau}, \quad G^{A}\left(\mathbf{r}_{1}-\mathbf{r}_{2}\right)=\int \frac{d^{3} p}{(2 \pi)^{3}} \frac{\exp \left[i \mathbf{p}\left(\mathbf{r}_{1}-\mathbf{r}_{2}\right)\right]}{\varepsilon-\xi-e \phi+i / 2 \tau}
$$

where $\phi$ is the local electric potential, which only slightly changes at their decay length $l_{i m p}$. The Keldysh Green's function, which contains information about the electron distribution, is given in equilibrium by

$$
G^{K}(\varepsilon)=\left[G^{A}(\varepsilon)-G^{R}(\varepsilon)\right] \tanh (\varepsilon / 2 T) .
$$

In the general case of a nonequilibrium dirty metal, it is conveniently expressed by means of the Dyson equation

$$
G^{K}\left(\varepsilon, \mathbf{r}_{1}, \mathbf{r}_{2}\right)=\frac{1}{2 \pi N_{F} \tau} \int d^{3} r^{\prime} G^{R}\left(\varepsilon, \mathbf{r}_{1}-\mathbf{r}^{\prime}\right) \bar{G}\left(\varepsilon, \mathbf{r}^{\prime}\right) G^{A}\left(\varepsilon, \mathbf{r}^{\prime}-\mathbf{r}_{2}\right)
$$

where $N_{F}$ is the Fermi density of states and

$$
\bar{G}(\varepsilon, \mathbf{r}) \equiv G^{K}(\varepsilon, \mathbf{r}, \mathbf{r}) .
$$

By setting $\mathbf{r}_{1}=\mathbf{r}_{2}$ in eqn. (8), one may obtain a diffusion equation for $\bar{G}$ in the form 20

$$
D_{0} \nabla^{2} \bar{G}=0
$$

where $D_{0}=v_{F}^{2} \tau / 3$ is the diffusion coefficient. Equation (8) suggests that all the diagrams for the expressions (住) - (6) are constructed of retarded and advanced Green's functions including Keldysh function vertices $\left(2 \pi N_{F} \tau\right)^{-1} \bar{G}$ and dressed with the random impurity potential correlators $\left(2 \pi N_{F} \tau\right)^{-1} \delta\left(\mathbf{r}_{1}-\mathbf{r}_{2}\right)$. Summing up an impurity-potential ladder between oppositely directed retarded and advanced Green's functions results in a diffuson

$$
\mathcal{D}\left(\mathbf{r}_{1}, \mathbf{r}_{2}, \omega\right)=\left\langle G^{R}\left(\mathbf{r}_{1}, \mathbf{r}_{2}, \varepsilon\right) G^{A}\left(\mathbf{r}_{2}, \mathbf{r}_{1}, \varepsilon+\omega\right)\right\rangle
$$

which obeys the equation 18

$$
\left[i \omega+D_{0} \frac{\partial^{2}}{\partial \mathbf{r}_{1}^{2}}\right] \mathcal{D}\left(\mathbf{r}_{1}, \mathbf{r}_{2}, \omega\right)=-2 \pi N_{F} \delta\left(\mathbf{r}_{1}-\mathbf{r}_{2}\right)
$$

and summing this ladder between the functions of the same direction results in a Cooperon, which obeys a similar equation. When performing the averaging, we retained only the terms of lowest order in $1 / p_{F} l_{i m p}$. This excludes any integrations over the momentum transferred by diffusons or Cooperons. In particular, any Cooperon impurity ladders as well as diffuson impurity ladders between different electron loops were omitted. This also implies that the localization corrections are neglected since they contain a small parameter $1 / p_{F} l_{i m p}$. 19 Hence the diagrams to be selected should consist of ordinary electron loops and diffusons connected in series and carrying the same momentum transfer $q_{t r}$. Our consideration is also restricted to the diffusion approximation, i.e. frequencies $\omega$ much smaller than the inverse elastic scattering time $\tau^{-1}$ and characteristic length scales much larger 


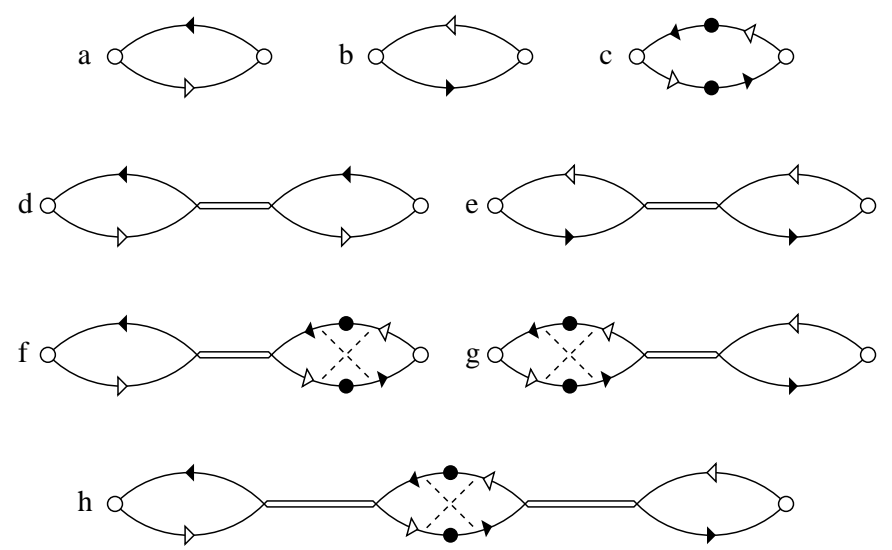

FIG. 2. Diagrams for $S_{\alpha \beta}^{(0)}$.

than $l_{i m p}$. This suggests that in the momentum representation, the diagrams selected be of lowest possible order or most singular in $q_{t r}$.

The diagrammatic expansion for $S_{\alpha \beta}^{(0)}$ is obtained by collecting diagrams of lowest order in $q_{t r}$ without interaction lines (see Fig. 2). Empty arrows denote single-electron advanced Green's functions, full arrows denote retarded Green's functions, full circles denote $\left(2 \pi N_{F} \tau\right)^{-1} \bar{G}$, and empty circles denote current vertices. The diagrams $a-c$ contain no diffusons. As $G^{R}$ and $G^{A}$ decay at a short length $l_{i m p}$, they form the term $\delta$-correlated in space, i.e. the analog of the bare correlator of extraneous currents in the Langevin approach.

Double lines in diagrams $2 d-2 h$ denote diffusons, which describe the propagation of electron density fluctuations and introduce a factor $q_{t r}^{-2}$. The left current ending to the diffuson in diagram $2 d$ reduces to is derivative $-2 \pi e N_{F} \tau D_{0} \partial / \partial r_{1 \alpha}$, and the left current ending to the diffuson in diagram $2 e$, to $2 \pi e N_{F} \tau D_{0} \partial / \partial r_{1 \alpha}$. The rest of diffuson endings are given by similar derivatives, their signs depending on the types of incoming and outgoing Green's functions, and result in a factor of $q_{t r}$. Physically, these derivatives reflect the fact that the current fluctuation is proportional to the density gradient. This is why diagrams $2 e$ and $2 d$ appear to be of zeroth order in $q_{t r}$ as well as $2 a-2 c$.

The advanced Green's functions change their type for retarded at the vertices including Keldysh functions $\bar{G}$. Wherever a diffuson is interrupted by a pair of such vertices (diagrams $2 f-2 h$ ), the diffuson frequency changes its sign and a Hikami box ${ }^{11}$ denoted by a dashed cross appears. This box is presented by the sum of the three diagrams shown in Fig. 3. Though each of them gives a contribution of zeroth order in $q_{t r}$, they cancel out each other so that the sum vanishes in a homogeneous metal at $q_{t r}=0$. A Hikami box with two diffuson vertices at $\mathbf{r}_{1}$ and $\mathbf{r}_{2}$ is given by the convolution of $\left(2 \pi N_{F} \tau\right)^{-1} \bar{G}\left(\mathbf{r}_{3}, \varepsilon\right)$ and $\left(2 \pi N_{F} \tau\right)^{-1} \bar{G}\left(\mathbf{r}_{4}, \varepsilon+\omega\right)$ with

$$
H_{0}\left(\mathbf{r}_{1}, \mathbf{r}_{2}, \mathbf{r}_{3}, \mathbf{r}_{4}\right)=2 \pi N_{F} \tau^{4} D_{0} \delta\left(\mathbf{r}_{1}-\mathbf{r}_{2}\right) \delta\left(\mathbf{r}_{1}-\mathbf{r}_{3}\right) \delta\left(\mathbf{r}_{1}-\mathbf{r}_{4}\right) \sum_{\gamma}\left(2 \frac{\partial}{\partial r_{1 \gamma}} \frac{\partial}{\partial r_{2 \gamma}}-\frac{\partial^{2}}{\partial r_{3 \gamma}^{2}}-\frac{\partial^{2}}{\partial r_{4 \gamma}^{2}}\right)
$$
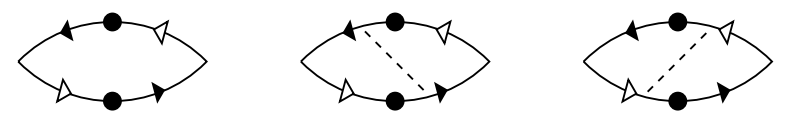

FIG. 3. Diagrams for the Hikami box $H_{0}$. Dashed lines represent the correlator of impurity potential $\left(2 \pi N_{F} \tau\right)^{-1} \delta\left(\mathbf{r}_{1}-\mathbf{r}_{2}\right)$. 
and the Hikami box with a current vertex at $\mathbf{r}_{1}$ and diffuson vertex at $\mathbf{r}_{2}$ is given by a convolution of the same quantities with

$$
H_{\alpha}\left(\mathbf{r}_{1}, \mathbf{r}_{2}, \mathbf{r}_{3}, \mathbf{r}_{4}\right)=4 \pi e N_{F} \tau^{4} D_{0} \delta\left(\mathbf{r}_{1}-\mathbf{r}_{2}\right) \delta\left(\mathbf{r}_{1}-\mathbf{r}_{3}\right) \delta\left(\mathbf{r}_{1}-\mathbf{r}_{4}\right) \frac{\partial}{\partial r_{2 \alpha}}
$$

Any diffuson vertex in a Hikami box introduces also a factor of $\left(2 \pi N_{F} \tau\right)^{-1}$. Correspondingly, these two types of boxes introduce factors of $q_{t r}^{2}$ and $q_{t r}$. Qualitatively, their role can be understood as follows: they convert local correlators of extraneous Langevin currents $\delta j^{\text {ext }}$ at some point into local fluctuations of charge density $\nabla \delta j^{e x t}$, whose propagation to points $\mathbf{r}_{1}$ and $\mathbf{r}_{2}$ is described by the diffusons.

As $\bar{G}$ remain finite at $\varepsilon \rightarrow \pm \infty$, integration of their products with respect to $\varepsilon$ would result in a divergency. To eliminate these divergencies, diagrams $f-h$ are combined with diagrams $d$ and $e$. Making use of eqn. (9) and the identity

$$
D_{0} \sum_{\gamma} \int d^{3} r^{\prime} \frac{\partial \mathcal{D}\left(\mathbf{r}_{1}, \mathbf{r}^{\prime},-\omega\right)}{\partial r_{\gamma}^{\prime}} \frac{\partial \mathcal{D}\left(\mathbf{r}_{2}, \mathbf{r}^{\prime}, \omega\right)}{\partial r_{\gamma}^{\prime}}=\pi N_{F}\left[\mathcal{D}\left(\mathbf{r}_{1}, \mathbf{r}_{2},-\omega\right)+\mathcal{D}\left(\mathbf{r}_{2}, \mathbf{r}_{1}, \omega\right)\right]
$$

the expression for $S_{\alpha \beta}^{(0)}$ may be presented in the form

$$
\begin{gathered}
S_{\alpha \beta}^{(0)}\left(\mathbf{r}_{1}, \mathbf{r}_{2}, \omega\right)=4 e^{2} N_{F} D_{0} \delta_{\alpha \beta} \delta\left(\mathbf{r}_{1}-\mathbf{r}_{2}\right) T_{N}\left(\mathbf{r}_{1}, \omega\right) \\
-\frac{2}{\pi} e^{2} D_{0}^{2} \frac{\partial^{2} \mathcal{D}\left(\mathbf{r}_{1}, \mathbf{r}_{2},-\omega\right)}{\partial r_{1 \alpha} \partial r_{2 \beta}} T_{N}\left(\mathbf{r}_{2}, \omega\right)-\frac{2}{\pi} e^{2} D_{0}^{2} \frac{\partial^{2} \mathcal{D}\left(\mathbf{r}_{2}, \mathbf{r}_{1}, \omega\right)}{\partial r_{1 \alpha} \partial r_{2 \beta}} T_{N}\left(\mathbf{r}_{1}, \omega\right) \\
+\frac{e^{2} D_{0}^{3}}{\pi^{2} N_{F}} \sum_{\gamma} \int d^{3} r^{\prime} \frac{\partial^{2} \mathcal{D}\left(\mathbf{r}_{1}, \mathbf{r}^{\prime},-\omega\right)}{\partial r_{1 \alpha} \partial r_{\gamma}^{\prime}} T_{N}\left(\mathbf{r}^{\prime}, \omega\right) \frac{\partial^{2} \mathcal{D}\left(\mathbf{r}_{2}, \mathbf{r}^{\prime}, \omega\right)}{\partial r_{\gamma}^{\prime} \partial r_{2 \beta}}
\end{gathered}
$$

where

$$
T_{N}(\mathbf{r}, \omega)=\frac{1}{4} \int d \varepsilon\left[1+\frac{1}{\left(2 \pi N_{F}\right)^{2}} \bar{G}(\mathbf{r}, \varepsilon) \bar{G}(\mathbf{r}, \varepsilon+\omega)\right]
$$

is the effective coordinate- and frequency-dependent noise temperature.

The most singular diagrams for the correlator $P_{\alpha}$ are shown in Fig. 4. They are very similar to the diagrams for $S_{\alpha \beta}^{(0)}$ except that the right-hand current endings are missing. For this reason, local contributions like $2 a-2 c$ are also missing, because they give a contribution of the order of $q_{t r}$,

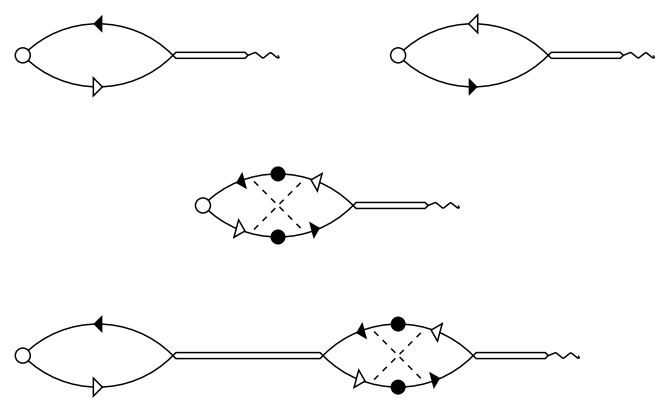

FIG. 4. Diagrams for $P_{\alpha}$. 


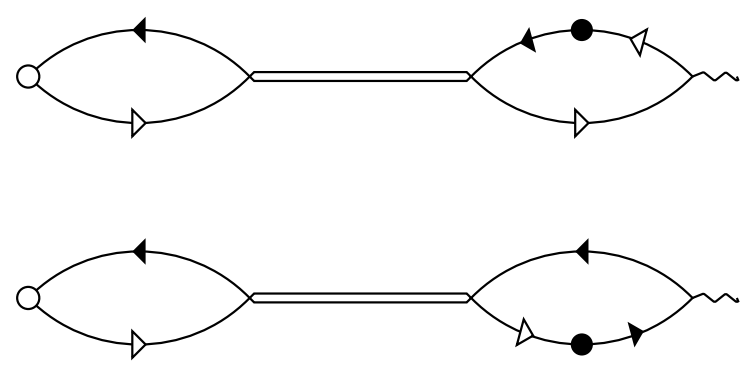

FIG. 5. Diagrams for $Q_{\alpha}$.

whereas the rest of diagrams are of the order of $q_{t r}^{-1}$. Combining the two first diagrams with the three last ones to eliminate the divergencies in integrals over $\varepsilon$ and making use of the identity (14), one obtains

$$
\begin{gathered}
P_{\alpha}\left(\mathbf{r}_{1}, \mathbf{r}_{2}, \omega\right)=\frac{4}{\pi} e D_{0} \frac{\partial \mathcal{D}\left(\mathbf{r}_{1}, \mathbf{r}_{2}, \omega\right)}{\partial r_{1 \alpha}} T_{N}\left(\mathbf{r}_{1}, \omega\right) \\
-\frac{2 e D_{0}^{2}}{\pi^{2} N_{F}} \sum_{\gamma} \int d^{3} r^{\prime} \frac{\partial^{2} \mathcal{D}\left(\mathbf{r}_{1}, \mathbf{r}^{\prime},-\omega\right)}{\partial r_{1 \alpha} \partial r_{\gamma}^{\prime}} T_{N}\left(\mathbf{r}^{\prime}, \omega\right) \frac{\partial \mathcal{D}\left(\mathbf{r}_{2}, \mathbf{r}^{\prime}, \omega\right)}{\partial r_{\gamma}^{\prime}}
\end{gathered}
$$

The most singular in $q_{t r}$ diagrams contributing to $Q_{\alpha}$ are shown in Fig. 5 . As this quantity contains only one Keldysh function, the diagrams for it are essentially different from those in Figs. 2 and 4 . The two terms in (6) give contributions of opposite signs yet with the energies in $\bar{G}$ shifted by $\omega$. Hence the integration over the energy gives

$$
Q_{\alpha}\left(\mathbf{r}_{1}, \mathbf{r}_{2}, \omega\right)=\frac{\omega}{\pi} e D_{0} \frac{\partial \mathcal{D}\left(\mathbf{r}_{1}, \mathbf{r}_{2},-\omega\right)}{\partial r_{1 \alpha}}
$$

Since $Q_{\alpha}$ is proportional to $\omega$, the contribution from the Coulomb interaction vanishes at $\omega=0$ and the noise in this case is essentially the same as for noninteracting electrons, much like it is in the semiclassical theory.

The potentials of screened interaction satisfy the equations

$$
\begin{aligned}
& V^{R}\left(\mathbf{r}_{1}, \mathbf{r}_{2}, \omega\right)=V_{0}\left(\mathbf{r}_{1}, \mathbf{r}_{2}\right)-i \int d^{3} \mathbf{r}^{\prime} \int d^{3} \mathbf{r}^{\prime \prime} V_{0}\left(\mathbf{r}_{1}, \mathbf{r}^{\prime}\right) \Pi^{R}\left(\mathbf{r}^{\prime}, \mathbf{r}^{\prime \prime}, \omega\right) V^{R}\left(\mathbf{r}^{\prime \prime}, \mathbf{r}_{2}, \omega\right), \\
& V^{A}\left(\mathbf{r}_{1}, \mathbf{r}_{2}, \omega\right)=V_{0}\left(\mathbf{r}_{1}, \mathbf{r}_{2}\right)-i \int d^{3} \mathbf{r}^{\prime} \int d^{3} \mathbf{r}^{\prime \prime} V_{0}\left(\mathbf{r}_{1}, \mathbf{r}^{\prime}\right) \Pi^{A}\left(\mathbf{r}^{\prime}, \mathbf{r}^{\prime \prime}, \omega\right) V^{A}\left(\mathbf{r}^{\prime \prime}, \mathbf{r}_{2}, \omega\right),
\end{aligned}
$$

and

$$
\begin{aligned}
V^{K}\left(\mathbf{r}_{1}, \mathbf{r}_{2}, \omega\right)=- & i \int d^{3} \mathbf{r}^{\prime} \int d^{3} \mathbf{r}^{\prime \prime} V_{0}\left(\mathbf{r}_{1}, \mathbf{r}^{\prime}\right)\left[\Pi^{R}\left(\mathbf{r}^{\prime}, \mathbf{r}^{\prime \prime}, \omega\right) V^{K}\left(\mathbf{r}^{\prime \prime}, \mathbf{r}_{2}, \omega\right)\right. \\
& \left.+\Pi^{K}\left(\mathbf{r}^{\prime}, \mathbf{r}^{\prime \prime}, \omega\right) V^{A}\left(\mathbf{r}^{\prime \prime}, \mathbf{r}_{2}, \omega\right)\right]
\end{aligned}
$$

where the bare interaction potential $V_{0}$ satisfies the Poisson equation

$$
\frac{\partial^{2}}{\partial \mathbf{r}_{1}^{2}} V_{0}\left(\mathbf{r}_{1}, \mathbf{r}_{2}\right)=-4 \pi e^{2} \delta\left(\mathbf{r}_{1}-\mathbf{r}_{2}\right)
$$


The polarization operators

$$
\begin{aligned}
& \Pi^{R}\left(\mathbf{r}_{1}, \mathbf{r}_{2}, \omega\right)=\frac{1}{4 \pi} \int d \varepsilon\left\langle G^{R}\left(\mathbf{r}_{1}, \mathbf{r}_{2}, \varepsilon+\omega\right) G^{K}\left(\mathbf{r}_{2}, \mathbf{r}_{1}, \varepsilon\right)+G^{K}\left(\mathbf{r}_{1}, \mathbf{r}_{2}, \varepsilon+\omega\right) G^{A}\left(\mathbf{r}_{2}, \mathbf{r}_{1}, \varepsilon\right)\right\rangle \\
& \Pi^{A}\left(\mathbf{r}_{1}, \mathbf{r}_{2}, \omega\right)=\frac{1}{4 \pi} \int d \varepsilon\left\langle G^{A}\left(\mathbf{r}_{1}, \mathbf{r}_{2}, \varepsilon+\omega\right) G^{K}\left(\mathbf{r}_{2}, \mathbf{r}_{1}, \varepsilon\right)+G^{K}\left(\mathbf{r}_{1}, \mathbf{r}_{2}, \varepsilon+\omega\right) G^{R}\left(\mathbf{r}_{2}, \mathbf{r}_{1}, \varepsilon\right)\right\rangle,
\end{aligned}
$$

describe the screening of bare potential (22), and the polarization operator

$$
\begin{aligned}
\Pi^{K}\left(\mathbf{r}_{1}, \mathbf{r}_{2}, \omega\right)=\frac{1}{4 \pi} \int d \varepsilon & \left\langle G^{R}\left(\mathbf{r}_{1}, \mathbf{r}_{2}, \varepsilon+\omega\right) G^{A}\left(\mathbf{r}_{2}, \mathbf{r}_{1}, \varepsilon\right)+G^{A}\left(\mathbf{r}_{1}, \mathbf{r}_{2}, \varepsilon+\omega\right) G^{R}\left(\mathbf{r}_{2}, \mathbf{r}_{1}, \varepsilon\right)\right. \\
& \left.+G^{K}\left(\mathbf{r}_{1}, \mathbf{r}_{2}, \varepsilon+\omega\right) G^{K}\left(\mathbf{r}_{2}, \mathbf{r}_{1}, \varepsilon\right)\right\rangle .
\end{aligned}
$$

actually presents the density - density correlator of noninteracting electrons.

Following Altshuler and Aronov, 22 one obtains expressions for $\Pi^{R}$ and $\Pi^{A}$ in the form

$$
\Pi^{R}\left(\mathbf{r}_{1}, \mathbf{r}_{2}, \omega\right)=\frac{i}{2 \pi} D_{0} \frac{\partial^{2} \mathcal{D}\left(\mathbf{r}_{1}, \mathbf{r}_{2},-\omega\right)}{\partial \mathbf{r}_{1}^{2}}, \quad \Pi^{A}\left(\mathbf{r}_{1}, \mathbf{r}_{2}, \omega\right)=\frac{i}{2 \pi} D_{0} \frac{\partial^{2} \mathcal{D}\left(\mathbf{r}_{1}, \mathbf{r}_{2}, \omega\right)}{\partial \mathbf{r}_{1}^{2}}
$$

For the particular case of an infinite conductor, solving the integral equations (19) and (20) with the kernels (26) gives the spatial Fourier transforms of $V^{R}$ and $V^{A}$ in the form

$$
V^{R}(q, \omega)=V^{A *}(q, \omega)=\frac{4 \pi e^{2}}{q^{2} \varepsilon(q, \omega)}, \quad \varepsilon(q, \omega)=1+\frac{4 \pi \sigma}{i \omega+D_{0} q^{2}},
$$

where $\sigma=e^{2} N_{F} D_{0}$ is the conductivity of the metal.

Making use of equations (19) and (20), the expression for $V^{K}$ may be recast in the form

$$
V^{K}\left(\mathbf{r}_{1}, \mathbf{r}_{2}, \omega\right)=-i \int d^{3} \mathbf{r}^{\prime} \int d^{3} \mathbf{r}^{\prime \prime} V^{R}\left(\mathbf{r}_{1}, \mathbf{r}^{\prime}, \omega\right) \Pi^{K}\left(\mathbf{r}^{\prime}, \mathbf{r}^{\prime \prime}, \omega\right) V^{A}\left(\mathbf{r}^{\prime \prime}, \mathbf{r}_{2}, \omega\right)
$$

This suggests that $V^{K}$ actually presents a correlator of fluctuating electric potentials caused by fluctuations of electron density.

The most singular in $q_{t r}$ diagrams for the density - density correlator $\Pi^{K}$ are shown in Fig. 6. Much like the diagrams for $P_{\alpha}$, these diagrams do not contain local terms of zeroth order in $q_{t r}$ since the leading contribution is proportional to $q_{t r}^{-2}$. The first two diagrams are combined with the third one using the identity (14), which results in an analytical expression

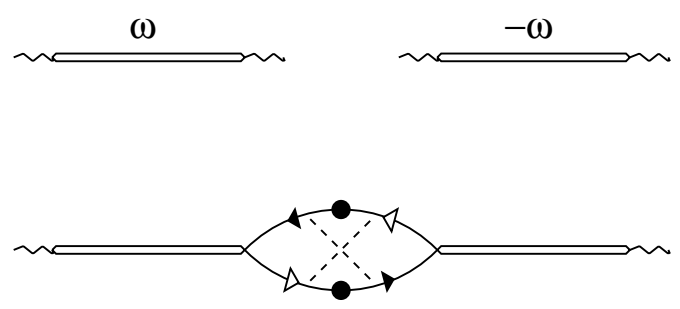

FIG. 6. Diagrams for $\Pi^{K}$. 


$$
\Pi^{K}\left(\mathbf{r}_{1}, \mathbf{r}_{2}, \omega\right)=\frac{D_{0}}{\pi^{2} N_{F}} \int d^{3} r^{\prime} \sum_{\gamma} \frac{\partial \mathcal{D}\left(\mathbf{r}_{1}, \mathbf{r}^{\prime},-\omega\right)}{\partial r_{\gamma}^{\prime}} T_{N}\left(\mathbf{r}^{\prime}, \omega\right) \frac{\partial \mathcal{D}\left(\mathbf{r}_{2}, \mathbf{r}^{\prime}, \omega\right)}{\partial r_{\gamma}^{\prime}}
$$

It should be noted that the terms involving the electron-electron interaction in (3) appear to be more singular in $q_{t r}$ than $S_{\alpha \beta}^{(0)}$. However they also contain excess powers of frequency $\omega$, which makes all the terms in (3) comparable in the diffusive limit.

In an equilibrium conductor with electric potential $\phi$ the Green's function $\bar{G}$ is of the form 20

$$
\bar{G}(\varepsilon)=-2 \pi i N_{F}\left[1-f_{0}(\varepsilon+e \phi)\right],
$$

where $f_{0}(\varepsilon)$ is the Fermi distribution function for a given temperature. Hence one may define the local distribution function in nonequilibrium metal via the relationship

$$
f(\mathbf{r}, \varepsilon)=\frac{1}{2}\left[1-\frac{i}{2 \pi N_{F}} \bar{G}(\mathbf{r}, \varepsilon)\right],
$$

so that it satisfies the standard diffusion equation and the boundary conditions for the semiclassical distribution function. This definition of $f$ is merely equivalent to the definition of Gramespacher and Büttiker in terms of distribution functions in electrodes and the injectivities of these electrodes. $\frac{0}{\text { In }}$ terms of $f$, the effective noise temperature $T_{N}$ may be written as

$$
T_{N}(\mathbf{r}, \omega)=\frac{1}{2} \int d \varepsilon\{f(\mathbf{r}, \varepsilon)[1-f(\mathbf{r}, \varepsilon+\omega)]+f(\mathbf{r}, \varepsilon+\omega)[1-f(\mathbf{r}, \varepsilon)]\} .
$$

In equilibrium, this expression reduces to

$$
T_{N}=\frac{1}{2} \omega \operatorname{coth}\left(\frac{\omega}{2 T}\right),
$$

which presents the standard factor in the Nyquist formula.

\section{QUANTUM BOLTZMANN - LANGEVIN SCHEME}

It is easily verified (see Appendix for details) that calculating the noise on the basis of Eqn. (3) results into the expressions for its spectral density identical to those given by the following quantum extension of the semiclassical Boltzmann - Langevin scheme. As well as in the semiclassical case, the fluctuation of current is formally described by a Langevin equation 14

$$
\delta \mathbf{j}=-D_{0} \frac{\partial}{\partial \mathbf{r}} \delta \rho+\sigma \delta \mathbf{E}+\delta \mathbf{j}^{\mathbf{e x t}},
$$

where the fluctuation of charge density $\delta \rho$ satisfies the equation

$$
\left(\frac{\partial}{\partial t}-D_{0} \nabla^{2}+4 \pi \sigma\right) \delta \rho=-\nabla \delta \mathbf{j}^{\mathbf{e x t}}
$$

the self-consistent fluctuation of electric potential obeys the Poisson equation

$$
\delta \mathbf{E}=-\nabla \delta \phi, \quad \nabla^{2} \delta \phi=-4 \pi \delta \rho,
$$

and the spectral density of extraneous currents $\delta j^{e x t}$ is given by

$$
\left\langle\left\langle\delta j_{\alpha}^{e x t}\left(\mathbf{r}_{1}\right) \delta j_{\beta}^{e x t}\left(\mathbf{r}_{\mathbf{2}}\right)\right\rangle\right\rangle_{\omega}=4 \sigma \delta_{\alpha \beta} \delta\left(\mathbf{r}_{1}-\mathbf{r}_{2}\right) T_{N}\left(\mathbf{r}_{1}, \omega\right)
$$


where $T_{N}$ is expressed in terms of the effective distribution function (30). It is noteworthy that the correlator of these currents is quantum, whereas the response to the extraneous currents remains semiclassical. The reason for this is that in dirty metals, the response functions are presented by diffusons and screened interaction lines connected in series, which describe classical propagation of fluctuations, and the interference corrections to them are small. It should be stressed that these interference corrections have nothing to do with the quantum noise, which results solely from the shifts in energy of the Keldysh functions that appear in pairs in ordinary electron loops and the Hikami boxes. For equilibrium systems, this noise is a universal feature 23 and does not depend on the presence of interference phenomena.

At $\omega \rightarrow 0$, equation (35) transforms into semiclassical Eqn. (8) of Ref.14. Hence it is not surprising that the low-frequency properties of quantum-coherent shot noise 6 . semiclassical approach 8

\section{QUANTUM NOISE IN A LONG CONTACT WITH SCREENING}

Now we apply this formalism to calculations of the zero-temperature shot noise in a long contact with strong external screening. Consider a contact of length $L$ and circular section with a diameter $2 r_{0} \ll L$ that connects two massive electrodes with a voltage drop $V$ between them. The third electrode presents a perfectly conducting grounded shielding of the contact, which is coaxial with it and isolated from its surface with a thin insulating film of thickness $\delta_{0}$ and dielectric constant $\varepsilon_{d}$ (see Fig. 7, inset). In this case, the distribution function (30) obeys a one-dimensional diffusion equation with the boundary conditions

$$
f(L / 2, \varepsilon)=f_{0}(\varepsilon-e V / 2), \quad f(L / 2, \varepsilon)=f_{0}(\varepsilon+e V / 2) .
$$

At zero temperature, the solution is of the form

$$
f(x, \epsilon)= \begin{cases}0, & \epsilon>e V / 2 \\ 1-x / L, & e V / 2>\epsilon>-e V / 2 \\ 1, & \epsilon<-e V / 2\end{cases}
$$

Hence

$$
T_{N}(x, \omega)=\omega / 2+\theta(e V-\omega)(e V-\omega) \frac{x}{L}\left(1-\frac{x}{L}\right) .
$$

Equations (32) - (34) were solved in 14 for the contact geometry in hand. Using the response of current at the left edge of the contact $\delta I$ to the extraneous current $\delta j$ obtained there, the spectral density of current fluctuations at one of the contact ends may be presented in the form

$$
S_{I}(\omega)=\frac{4}{R L} \int_{0}^{L} d x K(x, \omega) T_{N}(x, \omega),
$$

where $R$ is the resistance of the contact, $x$ is the longitudinal coordinate, and

$$
K(x, \omega)=2\left(\gamma_{\omega} L\right)^{2} \frac{\cosh \left[2 \gamma_{\omega}(L-x)\right]+\cos \left[2 \gamma_{\omega}(L-x)\right]}{\cosh \left(2 \gamma_{\omega} L\right)-\cos \left(2 \gamma_{\omega} L\right)},
$$

where $\gamma_{\omega}=\left(\omega \varepsilon_{d} / 4 \pi \sigma \delta_{0} r_{0}\right)^{1 / 2}$. At sufficiently high frequencies, the kernel $K$ exponentially decreases with $x$. This decrease has a simple physical explanation. At contact dimensions much larger than the 


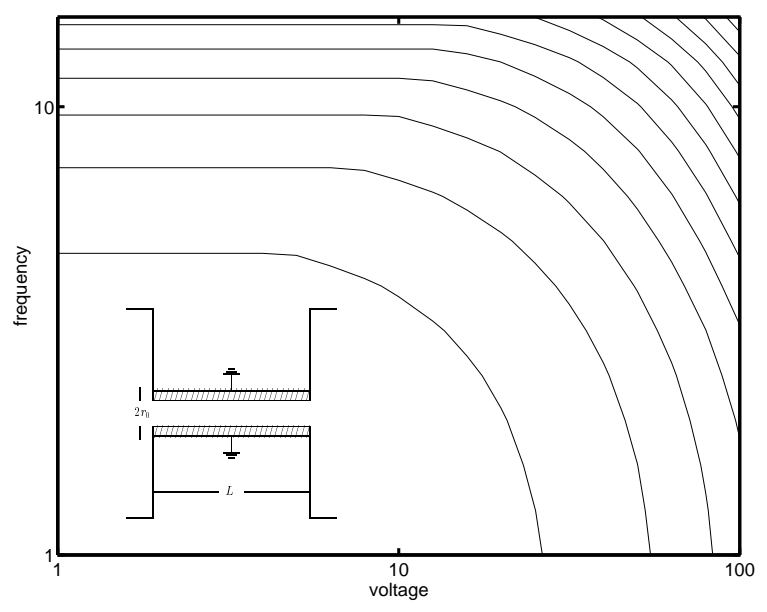

FIG. 7. Contour plots of $S_{I}$ vs. normalized voltage $e V L^{2} / 4 \pi \sigma \delta_{0} r_{0}$ and normalized frequency $\omega L^{2} / 4 \pi \sigma \delta_{0} r_{0}$. Inset shows the axial cross section of the contact.

Debye screening length, the local current fluctuations inside the contact described by correlator (35) induce the current fluctuations at the contact edges through the long-range fluctuations of electrical field. However at finite frequencies, the field-induced fluctuational charges are allowed to pile up at the outer surface of the contact. Hence the electric lines of force emerging from the middle points of the contact are intercepted by the screening electrode and do not reach the contact edges. Hence it is only the portions of the contact adjacent to its edges that contribute to the measurable noise.13.14 The integration gives

$$
\begin{gathered}
S_{I}(\omega)=2 \frac{\omega}{R} \gamma_{\omega} L \frac{\sinh \left(2 \gamma_{\omega} L\right)+\sin \left(2 \gamma_{\omega} L\right)}{\cosh \left(2 \gamma_{\omega} L\right)-\cos \left(2 \gamma_{\omega} L\right)} \\
+2 \theta(e V-\omega) \frac{e V-\omega}{R}\left[1-\frac{1}{\gamma_{\omega}} \frac{\sinh \left(2 \gamma_{\omega} L\right)-\sin \left(2 \gamma_{\omega} L\right)}{\cosh \left(2 \gamma_{\omega} L\right)-\cos \left(2 \gamma_{\omega} L\right)}\right] .
\end{gathered}
$$

In particular,

$$
S_{I}=2 \frac{\omega}{R}+\frac{2}{3} \theta(e V-\omega) \frac{e V-\omega}{R}
$$

at $\gamma_{\omega} L \ll 1$ and

$$
S_{I}=2 \gamma_{\omega} L \frac{\omega}{R}+2 \theta(e V-\omega) \frac{e V-\omega}{R}
$$

at $\gamma_{\omega} L \gg 1$. Note that in the latter case, the equilibrium quantum noise grows as $\omega^{3 / 2}$. In terms of the Nyquist formula $S_{I}=2 \omega \operatorname{coth}(\omega / 2 T) \mathcal{R} e Z^{-1}(\omega)$ this superlinear increase is due to the fact that the real part of inverse effective impedance $Z^{-1}(\omega)$ of the contact exhibits a square-root increase in this frequency range. Contour plots of $S_{I}$ vs. normalized voltage and normalized frequency are shown in Fig. 7. From this figure and Eqn. (43) it is readily seen that quantum noise (voltage-independent term) dominates over the shot noise (term proportional to voltage) even at $\omega \ll e V$ provided that $\omega$ is sufficiently large. The reason is that in this case the noise is caused by extraneous currents near the contact edges, where the distribution function only slightly differs from the equilibrium one. 1 In the opposite case of a short contact the standard expression (42) obtained in Ref.10 is valid for all frequencies. Recently, measurements of quantum shot noise were performed for mesoscopic diffusive contacts.24 25 Hence these results are of direct experimental interest. 


\section{CONCLUSION}

In summary, we have shown that in a phase-coherent diffusive metal, the high-frequency nonequilibrium noise may be described by semiclassical hydrodynamic Langevin equations with quantum sources. In long contacts with a strong external screening the crossover between the shot and quantum noise may take place at frequencies much lower than the voltage drop across the contact because the noise is dominated by the extreme contact regions, where the electron distribution is slightly affected by the applied voltage.

\section{ACKNOWLEDGMENTS}

The author is grateful to G. B. Lesovik for a stimulating discussion.

\section{APPENDIX}

The equivalence between the results of diagrammatic approach and of the proposed quantum extension of Boltzmann - Langevin method may be demonstrated as follows. By applying the Laplace operator to both sides of equations (19) and (20) and making use of the equation for the diffuson (11), one obtains that

$$
\begin{gathered}
\nabla^{2} V^{R}\left(\mathbf{r}_{1}, \mathbf{r}_{2}, \omega\right)=-4 \pi e^{2} \delta\left(\mathbf{r}_{1}-\mathbf{r}_{2}\right)+4 \pi e^{2} N_{F} V^{R}\left(\mathbf{r}_{1}, \mathbf{r}_{2}, \omega\right) \\
-2 i \omega e^{2} \int d^{3} \mathbf{r}^{\prime} \mathcal{D}\left(\mathbf{r}_{1}, \mathbf{r}^{\prime},-\omega\right) V^{R}\left(\mathbf{r}^{\prime}, \mathbf{r}_{2}, \omega\right) \\
\nabla^{2} V^{A}\left(\mathbf{r}_{1}, \mathbf{r}_{2}, \omega\right)=-4 \pi e^{2} \delta\left(\mathbf{r}_{1}-\mathbf{r}_{2}\right)+4 \pi e^{2} N_{F} V^{A}\left(\mathbf{r}_{1}, \mathbf{r}_{2}, \omega\right) \\
+2 i \omega e^{2} \int d^{3} \mathbf{r}^{\prime} \mathcal{D}\left(\mathbf{r}_{1}, \mathbf{r}^{\prime}, \omega\right) V^{A}\left(\mathbf{r}^{\prime}, \mathbf{r}_{2}, \omega\right)
\end{gathered}
$$

Using these expressions and the equation for diffuson (11), it is easily verified by direct substitution that the Fourier transform of equation (33) for the fluctuation $\delta \rho$ is satisfied by

$$
\delta \rho(\mathbf{r}, \omega)=\frac{1}{8 \pi^{2} e^{2} N_{F}} \frac{\partial^{2}}{\partial \mathbf{r}^{2}} \int d^{3} \mathbf{r}^{\prime} \int d^{3} \mathbf{r}^{\prime \prime} V^{A}\left(\mathbf{r}, \mathbf{r}^{\prime}, \omega\right) \mathcal{D}\left(\mathbf{r}^{\prime}, \mathbf{r}^{\prime \prime}, \omega\right) \nabla \delta \mathbf{j}^{e x t}\left(\mathbf{r}^{\prime \prime}\right) .
$$

In terms of the potential fluctuations $\delta \phi$, the fluctuation of the current (32) may be presented in the form

$$
\delta \mathbf{j}=-\nabla\left(D_{0} \delta \rho+\sigma \delta \phi\right)+\delta \mathbf{j}^{\text {ext }} .
$$

Since the screened potentials $V^{R(A)}$ and the potential fluctuation $\delta \phi$ obey the same boundary conditions, the Poisson equation for $\delta \phi(\underline{34})$ is easily solved to give

$$
\delta \phi(\mathbf{r}, \omega)=-\frac{1}{2 \pi e^{2} N_{F}} \int d^{3} \mathbf{r}^{\prime} \int d^{3} \mathbf{r}^{\prime \prime} V^{A}\left(\mathbf{r}, \mathbf{r}^{\prime}, \omega\right) \mathcal{D}\left(\mathbf{r}^{\prime}, \mathbf{r}^{\prime \prime}, \omega\right) \nabla \delta \mathbf{j}^{e x t}\left(\mathbf{r}^{\prime \prime}\right) .
$$

Making use of identity (45), the Laplace operator may be excluded from (46) and the expression (47) for $\delta \mathbf{j}$ may be recast in the form

$$
\delta j_{\alpha}(\mathbf{r}, \omega)=\delta j_{\alpha}^{e x t}(\mathbf{r}, \omega)+\int d^{3} \mathbf{r}^{\prime} \mathcal{K}_{\alpha}\left(\mathbf{r}, \mathbf{r}^{\prime}, \omega\right) \nabla \mathbf{j}^{\text {ext }}\left(\mathbf{r}^{\prime}\right),
$$


where

$$
\mathcal{K}_{\alpha}\left(\mathbf{r}_{1}, \mathbf{r}_{2}, \omega\right)=\frac{D_{0}}{2 \pi N_{F}} \frac{\partial \mathcal{D}\left(\mathbf{r}_{1}, \mathbf{r}_{2}, \omega\right)}{\partial r_{1 \alpha}}-\frac{i \omega D_{0}}{4 \pi^{2} N_{F}} \int d^{3} \mathbf{r}^{\prime} \int d^{3} \mathbf{r}^{\prime \prime} \frac{\partial \mathcal{D}\left(\mathbf{r}_{1}, \mathbf{r}^{\prime}, \omega\right)}{\partial r_{1 \alpha}} V^{A}\left(\mathbf{r}^{\prime}, \mathbf{r}^{\prime \prime}, \omega\right) \mathcal{D}\left(\mathbf{r}^{\prime \prime}, \mathbf{r}_{2}, \omega\right) .
$$

Integrating in (49) by parts, multiplying it by the corresponding equation for $\delta j_{\beta}^{e x t}\left(\mathbf{r}_{2},-\omega\right)$, and making use of the correlator (35), one obtains the spectral density of noise in the form

$$
\begin{gathered}
S_{\alpha \beta}\left(\mathbf{r}_{1}, \mathbf{r}_{2}, \omega\right)=4 \sigma \delta_{\alpha \beta} \delta\left(\mathbf{r}_{1}-\mathbf{r}_{2}\right) T_{N}\left(\mathbf{r}_{1}, \omega\right)-4 \sigma \frac{\partial \mathcal{K}_{\beta}\left(\mathbf{r}_{2}, \mathbf{r}_{1},-\omega\right)}{\partial r_{1 \alpha}} T_{N}\left(\mathbf{r}_{1}, \omega\right) \\
-4 \sigma \frac{\partial \mathcal{K}_{\alpha}\left(\mathbf{r}_{1}, \mathbf{r}_{2}, \omega\right)}{\partial r_{2 \beta}} T_{N}\left(\mathbf{r}_{2}, \omega\right)+4 \sigma \sum_{\gamma} \int d^{3} \mathbf{r}^{\prime} \frac{\partial \mathcal{K}_{\alpha}\left(\mathbf{r}_{1}, \mathbf{r}^{\prime}, \omega\right)}{\partial r_{\gamma}^{\prime}} \frac{\partial \mathcal{K}_{\beta}\left(\mathbf{r}_{2}, \mathbf{r}^{\prime},-\omega\right)}{\partial r_{\gamma}^{\prime}} T_{N}\left(\mathbf{r}^{\prime}, \omega\right) .
\end{gathered}
$$

Recalling that $V^{A}(-\omega)=V^{R}(\omega)$, it is easily seen that expression (51) is identical to (3) with $S_{\alpha \beta}^{(0)}$, $Q_{\alpha}, P_{\alpha}$, and $V^{K}$ given by (15), (18), (17), and (28), respectively.

${ }^{1}$ Sh. Kogan, Electronic Noise and Fluctuations in Solids, Cambridge University Press, 1996.

${ }^{2}$ M. J. M. de Jong and C. W. J. Beenakker, in Mesoscopic Electron Transport, NATO ASI, Ser. E, Vol. 345, edited by L. P. Kouwenhoven, G. Schön, and L. L. Sohn (Kluwer, Dordrecht, 1997).

${ }^{3}$ C. W. J. Beenakker and M. Büttiker, Phys. Rev. B 46, 1889 (1992).

${ }^{4}$ K. E. Nagaev, Phys. Lett. A 169, 103 (1992).

${ }^{5}$ R. Landauer, Phys. Rev. B 47, 16427 (1993); Ann. N. Y. Acad. Sc. 755, 417 (1995); Physica B 227, 156 (1996).

${ }^{6}$ Yu. V. Nazarov, Phys. Rev. Lett. 73, 134 (1994).

${ }^{7}$ Ya. M. Blanter and M. Büttiker, Phys. Rev. B 56, 2127 (1997).

${ }^{8}$ E. V. Sukhorukov and D. Loss, Phys. Rev. Lett. 80, 4959 (1998); Phys. Rev. B 59, 13054 (1999).

${ }^{9}$ T. Gramespacher and M. Büttiker, Phys. Rev. B 60, 2375 (1999).

${ }^{10}$ M. Büttiker, Phys. Rev. B 45, 3807 (1992).

${ }^{11}$ M. Büttiker, J. Math. Phys. 37, 4793 (1996).

12 M. H. Pedersen, S. A. van Langen, and M. Büttiker, Phys. Rev. B 57, 1838 (1998).

${ }^{13}$ Y. Naveh, D. V. Averin, and K. K. Likharev, Phys. Rev. Lett. 79, 3482 (1997).

${ }^{14}$ K. E. Nagaev, Phys. Rev. B 57, 4628 (1998).

${ }^{15}$ B. L. Altshuler, L. S. Levitov, and A. Yu. Yakovets, Pis'ma Zh. Eksp. Teor. Fiz., 59, 821 (1994) [JETP Lett. 59, 857 (1994)].

${ }^{16}$ Equations for correlation functions of Green's function fluctuations were recently derived by Sh. M. Kogan, Phys. Rev. A 44, 8072 (1991). In principle, these equations also could be used for our purpose. However their derivation involved a four-branch extension of Keldysh technique, and we prefer to use its standard version.

${ }^{17}$ L. V. Keldysh, Zh. Eksp. Teor. Fiz 47, 1515 (1964) [JETP 20, 1018 (1964)].

18 B. L. Altshuler and A. G. Aronov, in Electron-electron Interactions in Disordered Systems, edited by A. L. Efros and M. Pollak (North-Holland, Amsterdam, 1985), p. 1.

19 A. A. Abrikosov, Fundamentals of the Theory of Metals, North Holland, Amsterdam, 1988.

${ }^{20}$ A. I. Larkin and D. E. Khmelnitskii, Zh. Eksp. Teor. Fiz. 91, 1815 (1986) [JETP 64, 1075 (1986)].

${ }^{21}$ S. Hikami, Phys. Rev. B 24, 2671 (1981).

${ }^{22}$ B. L. Altshuler and A. G. Aronov, Zh. Eksp. Teor. Fiz., 75, 1610 (1978) [Sov. Phys. JETP 48, 812 (1978)].

${ }^{23}$ L. D. Landau and E. M. Lifshitz, Statistical Physics (Pergamon, London, 1958).

${ }^{24}$ R. J. Schoelkopf, P. J. Burke, A. A. Kozhevnikov, D. E. Prober, and M. J. Rooks, Phys. Rev. Lett. 78, 3370 (1997).

${ }^{25}$ R. J. Schoelkopf, A. A. Kozhevnikov, D. E. Prober, and M. J. Rooks, Phys. Rev. Lett. 80, 2437 (1998). 